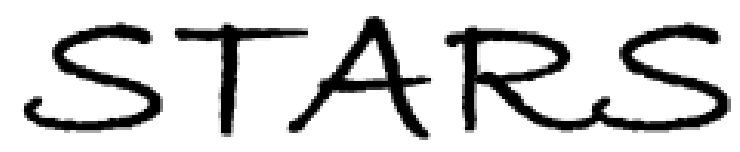

University of Central Florida

STARS

$1-1-1989$

\title{
Reconstruction Of Transform-Coded Images By Entropy Methods
}

Nicolaos S. Tzannes

University of Central Florida

Michael A. Tzannes

John Bodenschatz II

Find similar works at: https://stars.library.ucf.edu/facultybib1980

University of Central Florida Libraries http://library.ucf.edu

This Article is brought to you for free and open access by the Faculty Bibliography at STARS. It has been accepted for inclusion in Faculty Bibliography 1980 s by an authorized administrator of STARS. For more information, please contact STARS@ucf.edu.

\section{Recommended Citation}

Tzannes, Nicolaos S.; Tzannes, Michael A.; and Bodenschatz, John II, "Reconstruction Of TransformCoded Images By Entropy Methods" (1989). Faculty Bibliography 1980s. 853.

https://stars.library.ucf.edu/facultybib1980/853

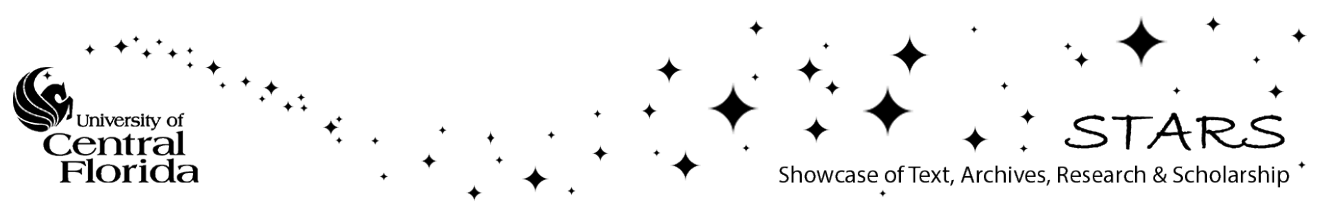




\section{Reconstruction of transform-coded images by entropy methods}

\author{
Nicolaos S. Tzannes, MEMBER SPIE \\ University of Central Florida \\ Department of Electrical Engineering \\ and Communication Sciences \\ Orlando, Florida 32816 \\ Michael A. Tzannes \\ Signatron, Inc. \\ Lexington, Massachusetts 02173 \\ John Bodenschatz II \\ National Security Agency \\ Washington, D.C. 20755
}

\begin{abstract}
A new, universal approach to reconstructing transform-coded images is proposed. The method views the images as a probability mass function (pmf), allowing the retained coefficients of a transform (KarhunenLoeve, discrete cosine, slant, etc.) to be thought of as averages of the basis functions over the pmf. This sets the stage for reconstructing the original images by using the maximum entropy principle (MEP) and the minimum relative entropy principle (MREP) with the retained coefficients as constraints in the extremizations. A formulation combining the two methods is also proposed, resulting in a reconstruction algorithm that is fast, proceeding in an iterative way using the estimate from each coefficient as a prior pmf for the next one via the MREP. The proposed approaches are illustrated with images compressed by discrete cosine transform coding, and the results are compared with standard reconstruction using the inverse discrete cosine transform.
\end{abstract}

Subject terms: image reconstruction; transform coding; relative entropy minimization.

Optical Engineering 28(3), 232-240 (March 1989).

\section{CONTENTS}

1. Background

2. Maximum entropy principle (MEP) and minimum relative entropy principle (MREP) reconstruction

2.1. MEP

3. Iterative algorithm for MEP and MREP

4. Applications

4.1. Example 1: the letter $O$

4.1.1. Coefficients retained: $5 \times 5$

4.1.2. Coefficients retained: $7 \times 7$

4.2. Example 2: the letters $O E$

4.3. Example 3: the Golden Gate Bridge

4.3.1. Block size: $8 \times 8$; coefficients retained: $2 \times 2$

4.3.2. Block size: $16 \times 16$; coefficients retained: $2 \times 2$

5. Conclusions

6. Acknowledgment

7. Appendix: convergence of the MEP-MREP algorithm

8. References

Paper 2534 received Feb. 18, 1988; revised manuscript received Dec. 28, 1988; accepted for publication Dec. 29, 1988. A preliminary version of this paper titled "Image reconstruction by entropy, relative entropy, and the DCT" was presented at the 1987 OSA Annual Meeting, Oct. 18-23, 1987, Rochester, N.Y.

(6) 1989 Society of Photo-Optical Instrumentation Engineers.

\section{BACKGROUND}

The proposed method of reconstructing images from their transform-coded from is based on the minimum relative entropy principle (MREP) and its subcase, the maximum entropy principle (MEP). Let us briefly outline these two principles.

Given two probability mass functions (pmf's) $f(j)$ and $p(j)$, their relative entropy (RE) is given by

$$
\sum_{j} f(j) \log \frac{f(j)}{p(j)} .
$$

This functional, introduced by Kullback, ${ }^{1}$ represents a "distance" between the two pmf's. It was originally named "discrimination of information or divergence," later "cross entropy" or "Kullback-Leibler number," and most recently "relative entropy." It can also be defined for probability density functions by replacing the sum with an integral.

In 1980 Shore and Johnson ${ }^{2}$ proposed that one could use the RE functional for estimating an unknown pmf $f(j)$, by minimizing it with respect to $f(j)$ and using a prior estimate or guess in place of $p(j)$. In applying this method, called the MREP, one usually has prior knowledge about $f(j)$ in the 
form of averages of functions $g_{n}(j)$ of the underlying random variable (rv) J. Such prior knowledge can be written as

$$
\sum_{j} g_{n}(j) f(j)=m_{n} \quad(n=0,1, \ldots, N)
$$

and can be incorporated into the minimization in the form of constraints. In most cases the functions are powers of $j$ and thus the averages are moments of the rv $J$.

It should be noted that if $p(j)$ is a constant (uniform pmf), then the RE becomes equivalent to the entropy of $J$ and the MREP reduces to the well-known MEP due to Jaynes. ${ }^{3}$

The mathematics of the minimization are straightforward and lead to the solution

$f(j)=p(j) \exp \left[-\lambda_{0}-\lambda_{1} g_{1}(j)-\ldots-\lambda_{n} g_{n}(j)\right]$,

where the $\lambda$ 's are Lagrangian multipliers evaluated by inserting this solution into the constraints of Eq. (2) and solving simlutaneously the resulting equations. If $p(j)$ is uniform, then the above solution reduces to the well-known MEP solution.

Both MREP and MEP enjoy the characteristic that their solutions satisfy the prior constraints but are unconstrained by information not explicitly present in the data.

Aside from pmf estimation, the above principles have been used in other areas, most notably in spectral estimation. Shore ${ }^{4}$ used the MREP in an indirect manner and Tzannes et al. ${ }^{5}$ in a direct one. Burg 6 was the first to employ the MEP in spectral estimation and others, too numerous to record here, followed.

The MREP and MEP have immediate extensions to twodimensional pmf's $f(j, k)$. The RE functional is

$$
\sum_{j} \sum_{k} f(j, k) \log \frac{f(j, k)}{p(j, k)}
$$

whereas the constraints have the form

$$
\sum_{j} \sum_{k} f(j, k) g_{n}(j, k)=M_{n}
$$

The MREP solution in terms of the Lagrangian multipliers is

$$
f(j, k)=p(j, k) \exp \left[-\lambda_{0}-\lambda_{1} g_{1}(j, k)-\ldots-\lambda_{n} g_{n}(j, k)\right],
$$

whereas the MEP solution is

$$
\mathrm{f}(\mathrm{j}, \mathrm{k})=\exp \left[-\lambda_{0}-\lambda_{1} \mathrm{~g}_{1}(\mathrm{j}, \mathrm{k})-\ldots-\lambda_{\mathrm{n}} \mathrm{g}_{\mathrm{n}}(\mathrm{j}, \mathrm{k})\right]
$$

\section{MEP AND MREP RECONSTRUCTION}

In most cases images are modeled deterministically, or taken to be a realization of a random field? The method we are about to present is based on the assumption that images can be modeled as a pmf of an underlying rv whose nature is of no consequence to the problem. Mathematically, a sampled image is indistinguishable from a two-dimensional pmf, since it is an everywhere positive function, and it can be easily normalized so that the sum of its pixel values becomes unity. References 8 and 9 advance even physical arguments for this idea, so there is no hesitation in adopting it here.
Let us consider an image whose uncompressed form is denoted by $f(j, k)$ for $0 \leq j, k \leq N-1$. Since this image is a pmf of a two-dimensional $\mathrm{rv} \mathbf{J} \times \mathbf{K}$, then the expression

$$
H(f)=-\sum_{j} \sum_{k} f(j, k) \log f(j, k)
$$

represents its entropy, and

$H(f, p)=\sum_{j} \sum_{k} f(j, k) \log \frac{f(j, k)}{p(j, k)}$

represents the relative entropy of $f(j, k)$ and $p(j, k)$ (another image). Let us compress this image by evaluating its discrete cosine transform (DCT) and retaining only a few of its terms for eventual transmission or storage. It should be remarked, however, that the DCT is taken only as an illustration and that the method is applicable to other types of compression schemes as well.

The DCT coefficients of $f(j, k)$ are given by 10

$F(m, n)=4 \sum_{j} \sum_{k} f(j, k) \cos \left[\frac{(2 j+1) \pi m}{2 N}\right] \cos \left[\frac{(2 k+1) \pi n}{2 N}\right]$.

Since the image is a pmf, these cofficients $F(m, n)$ can be thought of as averages of the functions

$\mathrm{g}_{\mathrm{mn}}(\mathrm{j}, \mathrm{k})=\cos \left[\frac{(2 \mathrm{j}+1) \pi \mathrm{m}}{2 \mathrm{~N}}\right] \cos \left[\frac{(2 \mathrm{k}+1) \pi \mathrm{n}}{2 \mathrm{~N}}\right]$.

Viewing the coefficients as averages of the above functions is, of course, the result of viewing the image as a pmf, and it is the key to what follows.

The reconstruction problem at the receiver (or upon recall, if stored) is to use the few retained $F(m, n)$ and estimate an approximation to the original image $f(j, k)$. The existing approach is to do so by using the inverse DCT (IDCT), setting all of the unknown coefficients equal to zero.

The proposed method, based on the above modeling of the image and interpretation of the coefficients, is to use the MEP and MREP as described in the following subsections.

\subsection{MEP}

Reconstruct the image by maximizing the entropy of $f(j, k)$ subject to constraints that reflect the known, retained coefficients. The general solution will yield an estimate of the form of Eq. (7), with the g's given by Eq. (11). If, for example, only one coefficient, the $F(1,1)$, is a vailable, then the maximization is subject to the single constraint

$4 \sum_{j} \sum_{k} f(j, k) \cos \left[\frac{(2 j+1) \pi}{2 N}\right] \cos \left[\frac{(2 k+1) \pi}{2 N}\right]=F(1,1)$,

and thus the solution will yield the estimate

$f(j, k)=\exp \left\{-\lambda_{1} \cos \left[\frac{(2 j+1) \pi}{2 N}\right] \cos \left[\frac{(2 k+1) \pi}{2 N}\right]\right\}$,

with $\lambda_{1}$ being a Lagrangian multiplier calculated by substituting the above solution into Eq. (12). 
In most practical problems the retained coefficients are numerous, although fewer than the original number of pixels in the image. The Lagrangian multipliers will be as many as the retained coefficients and will be calculated by solving the constraints simultaneously.

The idea of viewing the image as a pmf and using the MEP for its reconstruction has also been applied successfully to moment-coded images by Tzannes and Jonnard. ${ }^{11}$ Other investigations have also used the pmf modeling and MEP for other problems, such as, for example, Frieden ${ }^{9}$ and Daniell and Gull ${ }^{12}$ in problems of image enhancement in the presence of noise.

\subsection{MREP}

Let us now assume that besides the $F(m, n)$ 's, a prior estimate (or guess) $p(j, k)$ is available for the image $f(j, k)$. In this case the image can be reconstructed by minimizing the $R E$ of $f(j, k)$ and $p(j, k)$ subject to the same constraints as in the MEP. The general MREP solution yields an estimate of the form of Eq. (6), with the g's given by Eq. (11). The MREP yields a better estimate than the MEP since it takes into account the additional information provided by the prior estimate $p(j, k)$.

The MREP has also been used by Shore ${ }^{13}$ in problems of image enhancement. However, to the best of the author's knowledge, the present paper represents the first use of MEP and MREP in problems of reconstructing transform-coded images.

The proposed MEP and MREP methods are applicable, of course, to all types of transform coding (Karhunen-Loeve, discrete Fourier, Walsh-Hadamar, slant, Haar, etc.) since the retained coefficients in all of these transforms can be viewed as averages of the basis functions over the image (a pmf). They are also applicable to other types of compression methods (pyramid, for example) as long as the retained or reduced values can be viewed as averages or weighted averages of the whole or a portion of the image.

Both methods involve the solution of a system of many nonlinear equations for the Lagrangian multipliers. Nontrivial numerical methods such as Newton-Raphson procedures, which are usually employed, often become inefficient and numerically sensitive as the number of constraints is increased. For these reasons a new iterative algorithm for the solution of MEP and MREP is proposed below. It is theoretically justified in the appendix.

\section{ITERATIVE ALGORITHM FOR MEP AND MREP}

Since the MEP is a special case of the MREP [with $p(j, k)$ equal to unity], we present the proposed algorithm in the context of the MREP.

The whole idea behind the algorithm is to use one constraint at a time, arrive at an estimate, and then use this estimate as a prior $p(j, k)$ for the use of the next constraint.

Denoting the original image by $f(j, k)$ and the retained coefficients by $F(m, n)$, the algorithm proceeds as follows:

Step 1. The RE between $\mathrm{f}(\mathrm{j}, \mathrm{k})$ and $\mathrm{p}(\mathrm{j}, \mathrm{k})$ is minimized with only the first coefficient $F(0,0)$ as a constraint. This leads to the solution

$\mathrm{f}_{00}(\mathrm{j}, \mathrm{k})=\mathrm{p}(\mathrm{j}, \mathrm{k}) \exp \left(-\lambda_{0}\right)$

with the single Lagrangian multiplier easily calculable from the single constraint. This solution is thought of as the first reconstruction to the original image $f(j, k)$.

Step 2. The above first reconstruction is next used as a prior estimate for the unknown image $f(j, k)$, and a second reconstruction is obtained by minimizing the $\mathrm{RE}$ between $f(j, k)$ and $f_{00}(j, k)$, subject to the constraint

$\sum_{j} \sum_{k} f(j, k) \cos \left[\frac{(2 k+1) n}{2 N}\right]=F(0,1)$,

which reflects knowledge of the second coefficient $F(0,1)$. The solution and second reconstruction is given by

$f_{01}(j, k)=f_{00}(j, k) \exp \left\{-\lambda_{1} \cos \left[\frac{(2 k+1) \pi}{2 N}\right]\right\}$,

with the single Lagrangian multiplier easily calculable from the single constraint of Eq. (15).

Step 3. The solution Eq. (16) is used as a prior in minimizing the $R E$ of $f(j, k)$ and the second reconstruction, subject to the third constraint reflecting the coefficient $F(1,0)$. This leads to the third reconstruction, given by

$\mathrm{f}_{10}(\mathrm{j}, \mathrm{k})=\mathrm{f}_{01}(\mathrm{j}, \mathrm{k}) \exp \left\{-\lambda_{2} \cos \left[\frac{(2 \mathrm{j}+1) \pi}{2 \mathrm{~N}}\right]\right\}$,

with the single Lagrangian multiplier easily calculable from the third constraint.

Step 4. Repeat the procedure until all of the constraints are used up. The last reconstruction is taken as the solution of the MREP method.

The general solution of this algorithm can be written as

$f^{(n)}(j, k)=f^{(n-1)}(j, k) \exp \left[-\lambda_{n} g(j, k)\right]$,

where $f^{(n)}(j, k)$ is the $n$th reconstruction, $f^{(n-1)}(j, k)$ is the $(\mathrm{n}-\mathrm{l})$ th reconstruction, $\mathrm{g}(\mathrm{j}, \mathrm{k})$ is the $\mathrm{n}$ th basis element whose average over the image is used as a constraint, and $\lambda_{n}$ is the Lagrangian multiplier that goes with the $\mathrm{n}$ th constraint.

The solution obtained by this algorithm is only an approximation to the solution obtained by the regular use of the MREP (or the MEP if no initial guess is available). Only the final constraint is certain to be satisfied; the others are reflected in the form of priors. For this reason the solution obtained by a first sweep through the constraints is taken to be the first "iteration" to the desired MREP (or MEP) solution. Further passages through the constraints are usually necessary, although in the attempted examples two passages seem to suffice. However, even if more than two passages are necessary, much less computer time is needed than for the regular MREP.

The passage is, of course, stopped when successive iterations lead to solutions whose distance (mean-squared error) is small. In many cases this occurs much faster than in the normal MREP approach.

The above iterative method is also applicable to other areas in which MEP or MREP can be employed. It has recently been applied with good results to $\mathrm{pmf}^{14}$ and power spectrum estimation. ${ }^{15}$ It can also be applied to any other single or multidimensional data that are nonnegative or where the addition of a bias to make them nonnegative does not alter the nature of the problem. It appears to be also well suited for problems of "progressive" image reconstruction, ${ }^{16}$ especially 


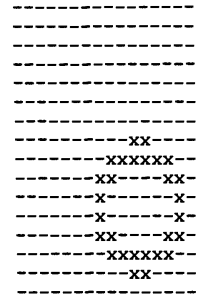

(a)

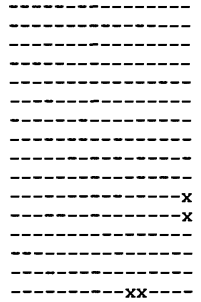

(b)

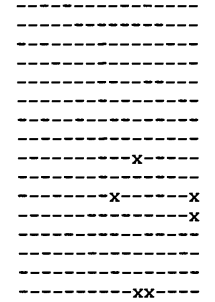

(c)

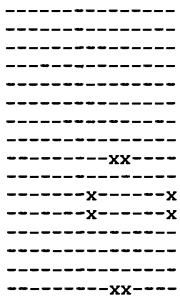

(d)

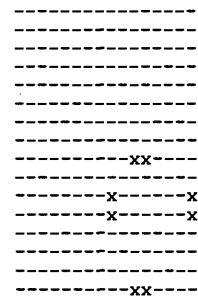

(e)
Fig. 1. Reconstructions of the letter $O$ with $5 \times 5$ coefficients retained. (a) Original image. (b) IDCT. (c) Regular MEP. (d) First iteration of MEP-MREP. (e) Second iteration of MEP-MREP.

when such reconstruction is performed in remote terminals with weak computing capabilities.

\section{APPLICATIONS}

In this section we present three examples of reconstructing DCT-coded images: by the use of the IDCT, the regular MEP, and the iterative MEP algorithm, which uses MREP. This third algorithm is referred to as the MEP-MREP.

\subsection{Example 1: the letter 0}

The original image, shown in Fig. 1(a), is a $16 \times 16$ pixel image with values of one (dashes) and two (x's), representing a noncentered letter $\mathbf{O}$.

The DCT was calculated for this image, a $16 \times 16$ matrix of coefficients. Next, subsets $n \times n$ of this matrix were retained, and reconstructions were obtained using the IDCT, the regular MEP, and the iterative MEP-MREP. Selected examples of $n \times n$ retained coefficients are discussed below. The reconstructions resembled the $O$ after the retained set reached $5 \times 5$.

\subsubsection{Coefficients retained: $5 \times 5$}

This represents a data compression ratio of 10.24 . Figure 1(b) shows the reconstruction by IDCT after the values were thresholded at 1.5 (i.e., values higher than or equal to 1.5 were made 2). The mean-squared error (MSE) (before thresholding) between the original and the reconstruction was 4.127. Figure 1(c) shows the reconstruction using the regular MEP, solving 25 constraint equations simultaneously. The MSE was 4.117. Visually, the reconstruction is better than that obtained by IDCT.

Figure 1(d) shows the result of reconstructing the image with one iteration of the MEP-MREP. This is defined as one passage through the 25 constraints, plus a single passage through the $\mathrm{F}(0,0)$ coefficient for renormalization purposes. The MSE was 4.121. Interestingly, this reconstruction is the best visually (so far), and it has lower MSE than the IDCT. Figure $1(\mathrm{e})$ is the reconstruction by iterative MEP-MREP

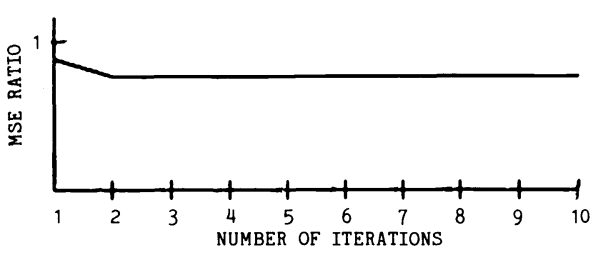

Fig. 2. Letter 0 . Graph of MEP-MREP/IDCT MSE vs number of iterations with $5 \times 5$ coefficients retained.

TABLE I. Results of reconstruction of the letter 0 .

\begin{tabular}{|c|c|c|}
\hline $\begin{array}{l}\text { Coefficients } \\
\text { retained } \\
(n \times n)\end{array}$ & Method & MSE \\
\hline $3 \times 3$ & $\begin{array}{l}\text { IDCT } \\
\text { Regular MEP } \\
\text { MEP-MREP } 1 \text { iteration } \\
2 \text { iterations } \\
3 \text { iterations }\end{array}$ & $\begin{array}{l}4.479 \\
4.459 \\
4.459 \\
4.459 \\
4.459\end{array}$ \\
\hline $5 \times 5$ & $\begin{array}{l}\text { IDCT } \\
\text { Regular MEP } \\
\text { MEP-MREP } 1 \text { iteration } \\
2 \text { iterations } \\
3 \text { iterations }\end{array}$ & $\begin{array}{l}4.127 \\
4.117 \\
4.121 \\
4.117 \\
4.117\end{array}$ \\
\hline $7 \times 7$ & $\begin{array}{l}\text { IDCT } \\
\text { Regular MEP } \\
\text { MEP-MREP } 1 \text { iteration } \\
2 \text { iterations } \\
3 \text { iterations }\end{array}$ & $\begin{array}{l}3.398 \\
3.211 \\
3.236 \\
3.211 \\
3.211\end{array}$ \\
\hline $9 \times 9$ & $\begin{array}{l}\text { IDCT } \\
\text { Regular MEP } \\
\text { MEP-MREP } 1 \text { iteration } \\
2 \text { iterations } \\
3 \text { iterations }\end{array}$ & $\begin{array}{l}3.026 \\
2.758 \\
2.803 \\
2.754 \\
2.754\end{array}$ \\
\hline $11 \times 11$ & $\begin{array}{l}\text { IDCT } \\
\text { Regular MEP } \\
\text { MEP-MREP } 1 \text { iteration } \\
2 \text { iterations } \\
3 \text { iterations }\end{array}$ & $\begin{array}{l}2.008 \\
1.655 \\
1.807 \\
1.655 \\
1.655\end{array}$ \\
\hline $13 \times 13$ & $\begin{array}{l}\text { IDCT } \\
\text { Regular MEP } \\
\text { MEP-MREP } 1 \text { iteration } \\
2 \text { iterations } \\
3 \text { iterations }\end{array}$ & $\begin{array}{l}1.703 \\
1.351 \\
1.578 \\
1.354 \\
1.351\end{array}$ \\
\hline
\end{tabular}

with two iterations. The MSE is reduced to 4.117, i.e., equal to the MSE of the regular MEP.

Figure 2 is a graph of the ratio of the iterative MEP-MREP MSE to the IDCT MSE versus the number of iterations. It is clear that two iterations are sufficient.

The inevitable conclusions are that, in this case, the IDCT is inferior to both MEP methods and that the MEP-MREP converges rapidly.

The results of these reconstructions as well as reconstructions by other $n \times n$ sets (not discussed) are summarized in Table I.

\subsubsection{Coefficients retained: $7 \times 7$}

This represents a data compression ratio of 5.22. Figure 3(a) shows the IDCT reconstruction after the values were thresholded at 1.5. The MSE was 3.398. Figure 3(b) shows the regular MEP reconstruction with the same threshold. The 


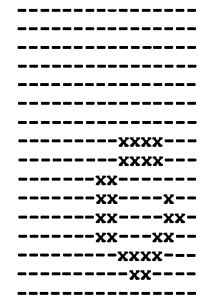

(a)

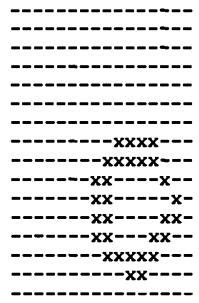

(b)

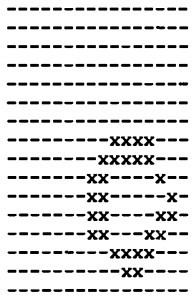

(d)

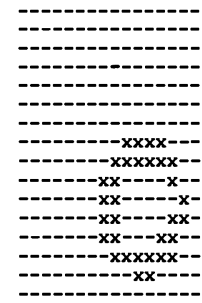

(c)

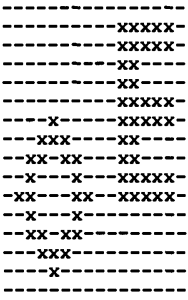

(a)

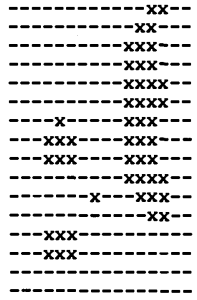

(b)

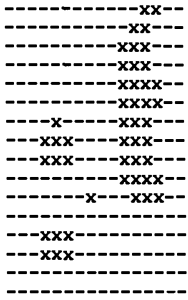

(c)

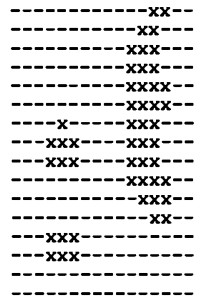

(d)

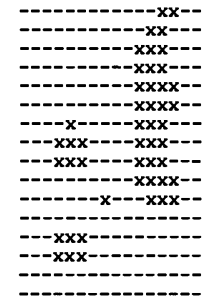

(e)
Fig. 3. Reconstructions of the letter 0 with $7 \times 7$ coefficients retained. (a) IDCT. (b) Regular MEP. (c) First iteration of MEP-MREP. (d) Second iteration of MEP-MREP.

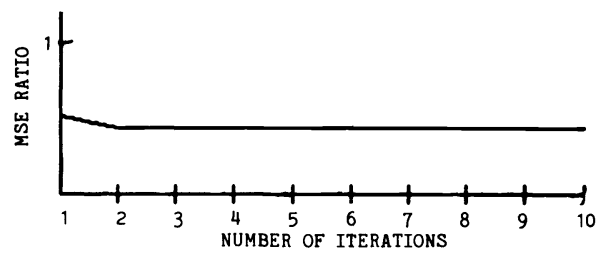

Fig. 4. Letter $O$. Graph of MEP-MREP/IDCT MSE vs number of iterations with $7 \times 7$ coefficients retained.

MSE was 3.211. This reconstruction is visually better than the IDCT reconstruction.

Figure 3(c) shows the first iteration obtained with the iterative MEP-MREP algorithm. The MSE was 3.236. This reconstruction is also visually better than the IDCT reconstruction. Figure 3(d) shows the second iteration of the proposed algorithm. The MSE was reduced to 3.211, i.e., equal to the regular MEP. There is no visual improvement.

Figure 4 is a graph of the ratio of the iterative MEP-MREP MSE to the IDCT MSE versus the number of iterations. The iterative algorithm converges with two passes through the constraints.

The conclusions are that the regular MEP and the iterative MEP-MREP algorithm perform better than the IDCT and that the MEP-MREP converges with two iterations.

The results are summarized in Table I. The situation was same with other sets of retained coefficients, as can be seen in this table.

\subsection{Example 2: the letters $\mathrm{OE}$}

The original image of "ones" and "twos" (dashes and x's, respectively) is shown in Fig. 5(a). The reconstructed images did not resemble the original image until the set of retained coefficients became $7 \times 7$. Figure 5(b) shows the IDCT reconstruction for this set of retained coefficients. Figure 5(c) shows the regular MEP reconstruction, and Figs. 5(d) and 5(e) show the reconstructions of the MEP-MREP with 1 and 2 itera-
Fig. 5. Reconstructions of letters $\mathrm{OE}$ with $7 \times 7$ coefficients retained. (a) Original image. (b) IDCT. (c) Regular MEP. (d) First iteration of MEP-MREP. (e) Second iteration of MEP-MREP.

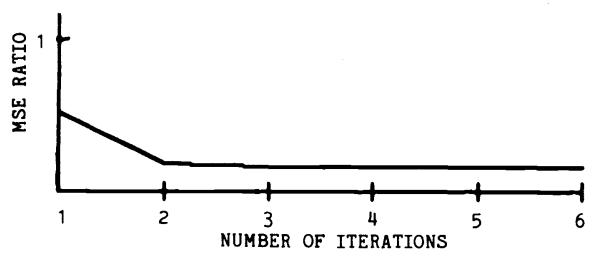

Fig. 6. Letters OE. Graph of MEP-MREP/IDCT MSE vs number of iterations with $7 \times 7$ coefficients retained.

tions, respectively. Figure 6 is a graph of the ratio of the iterative MEP-MREP MSE to the IDCT MSE versus the number of iterations. Table II summarizes the results for these and several other sets of $n \times n$ retained coefficients.

In this example the results were visually similar to each other. However, the MSEs followed the same pattern as in the previous example. The iterative algorithm converges with two iterations.

\subsection{Example 3: the Golden Gate Bridge}

A Gould 84000 image processor was employed for this example. The results were printed out on an HP Laserjet printer.

The original picture of the Golden Gate bridge, shown in Fig. 7, has $512 \times 512$ pixels and 256 gray levels. The DCT was performed on various sizes of blocks of pixels, as were the reconstructions.

\subsubsection{Block size: $8 \times 8$; coefficients retained: $2 \times 2$}

The compression ratio in this case is 16 . Figure 8 (a) shows the IDCT reconstruction. The MSE was 3419.339. Figure 8(b) shows the regular MEP reconstruction. The MSE was 3409.142. Figure 8(c) shows the first iteration of the MEPMREP algorithm. The MSE was 3414.039. Figure 8(d) shows the second iteration of the MEP-MREP algorithm. The MSE was 3409.157 . The third iteration was the same. 


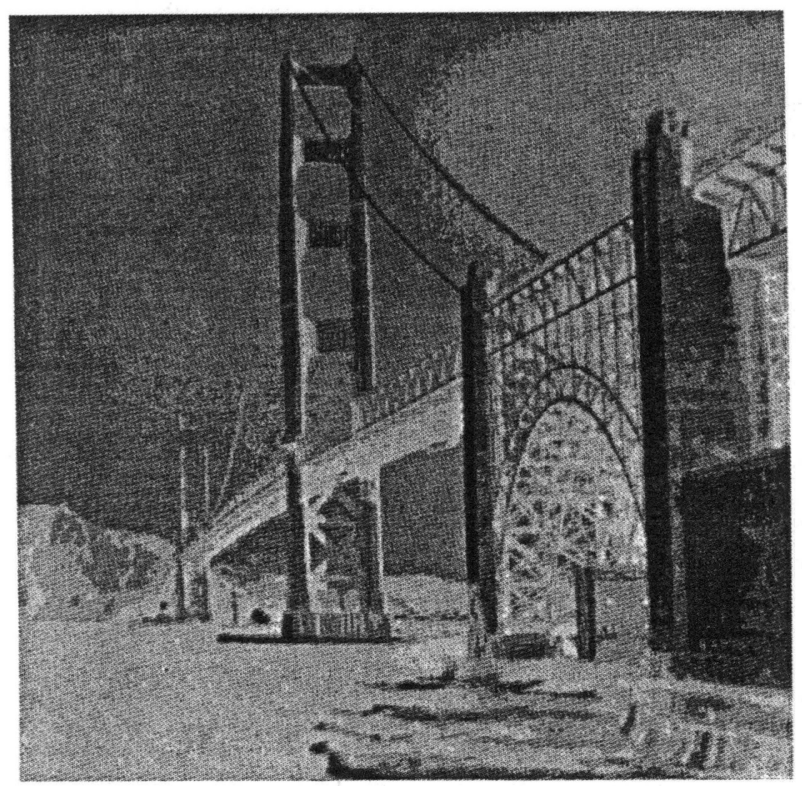

Fig. 7. The original picture of the Golden Gate Bridge.

TABLE II. Results of reconstruction of the letter OE.

\begin{tabular}{|c|c|c|}
\hline $\begin{array}{l}\text { Coefficients } \\
\text { retained } \\
(n \times n) \\
\end{array}$ & Method & MSE \\
\hline $3 \times 3$ & $\begin{array}{l}\text { IDCT } \\
\text { Regular MEP } \\
\text { MEP-MREP } 1 \text { iteration } \\
2 \text { iterations } \\
3 \text { iterations }\end{array}$ & $\begin{array}{l}6.268 \\
6.267 \\
6.270 \\
6.267 \\
6.267\end{array}$ \\
\hline $5 \times 5$ & $\begin{array}{l}\text { IDCT } \\
\text { Regular MEP } \\
\text { MEP-MREP } 1 \text { iteration } \\
2 \text { iterations } \\
3 \text { iterations }\end{array}$ & $\begin{array}{l}5.469 \\
5.468 \\
5.475 \\
5.469 \\
5.468\end{array}$ \\
\hline $7 \times 7$ & $\begin{array}{l}\text { IDCT } \\
\text { Regular MEP } \\
\text { MEP-MREP } 1 \text { iteration } \\
2 \text { iterations } \\
3 \text { iterations }\end{array}$ & $\begin{array}{l}4.880 \\
4.840 \\
4.856 \\
4.840 \\
4.840\end{array}$ \\
\hline $9 \times 9$ & $\begin{array}{l}\text { IDCT } \\
\text { Regular MEP } \\
\text { MEP-MREP } 1 \text { iteration } \\
2 \text { iterations } \\
3 \text { iterations }\end{array}$ & $\begin{array}{l}3.299 \\
3.053 \\
3.144 \\
3.052 \\
3.052\end{array}$ \\
\hline $11 \times 11$ & $\begin{array}{l}\text { IDCT } \\
\text { Regular MEP } \\
\text { MEP-MREP } 1 \text { iteration } \\
2 \text { iterations } \\
3 \text { iterations }\end{array}$ & $\begin{array}{l}2.428 \\
2.198 \\
2.338 \\
2.199 \\
2.198\end{array}$ \\
\hline $13 \times 13$ & $\begin{array}{l}\text { IDCT } \\
\text { Regular MEP } \\
\text { MEP-MREP } 1 \text { iteration } \\
2 \text { iterations } \\
3 \text { iterations }\end{array}$ & $\begin{array}{l}1.857 \\
1.645 \\
1.865 \\
1.650 \\
1.646\end{array}$ \\
\hline
\end{tabular}

\subsubsection{Block size: $16 \times 16$; coefficients retained: $2 \times 2$}

The compression ratio in this case is 64 . Figure 9(a) shows the IDCT reconstruction. The MSE was 5300.152. Figure 9(b) shows the regular MEP reconstruction. The MSE was 5280.852. Figure 9(c) shows the first iteration of the MEP-
MREP algorithm. The MSE was 5285.891. Figure 9(d) shows the second iteration of the MEP-MREP algorithm. The MSE was 5281.043. The third iteration was the same as the regular MEP.

Other combinations of block sizes and retained coefficient sizes produced similar results. Visually, the differences between the IDCT and the proposed methods were unnoticeable. The MSEs were slightly less for the proposed methods. The iterative MEP-MREP algorithm appeared to converge with two passes and in some cases, with three.

\section{CONCLUSIONS}

The proposed MEP and MREP methodologies for reconstructing transform-coded images seem legitimate and mathematically universal, i.e., a pplicable to all transform coding. In this paper they were compared with DCT coding since such coding is approximately close to the ideal (Karhunen-Loeve) and not data dependent. In the examples attempted, the regular MEP approach performed better than (or as well as) the IDCT with respect to MSEs.

The proposed MEP-MREP iterative algorithm appears to converge very rapidly. Even the first iteration was usually better than the IDCT reconstruction. It is free of the slowness and convergency problems encountered in the regular MEP or MREP.

\section{ACKNOWLEDGMENT}

This work was partially supported by a grant from the Center for Electro-Optics and Lasers (CREOL) of the University of Central Florida.

\section{APPENDIX: CONVERGENCE OF THE MEP-MREP ALGORITHM}

Let us consider a MREP problem with prior $\mathrm{p}(\mathrm{j})$ and only two constraints,

$$
\begin{aligned}
& \sum_{j} g_{0}(j) f(j)=m_{0}, \\
& \sum_{j} g_{1}(j) f(j)=m_{1},
\end{aligned}
$$

where $f(j)$ is the true pmf being sought. The consideration of only two constraints makes the concepts clearer.

Under the proposed MEP-MREP algorithm the constraints are treated one at a time.

Considering only the constraint of Eq. (19), the minimization of the RE between $f(j)$ and $p(j)$ leads to the solution

$f_{0}(j)=p(j) \exp \left[-\lambda_{0} g_{0}(j)\right]$.

The single Lagrangian multiplier $\lambda_{0}$ is next calculated from the constraint of Eq. (19), and this $\mathrm{f}_{0}(\mathrm{j})$ is considered the new prior.

The RE between $f(j)$ and this new prior $f_{0}(j)$ is next minimized, subject to the second constraint, Eq. (20). The solution is denoted by $f_{1}(j)$ and has the form

$$
\begin{aligned}
f_{1}(j) & =f_{0}(j) \exp \left[-\lambda_{1} g_{1}(j)\right] \\
& =p(j) \exp \left[-\lambda_{0} g_{0}(j)-\lambda_{1} g_{1}(j)\right] .
\end{aligned}
$$




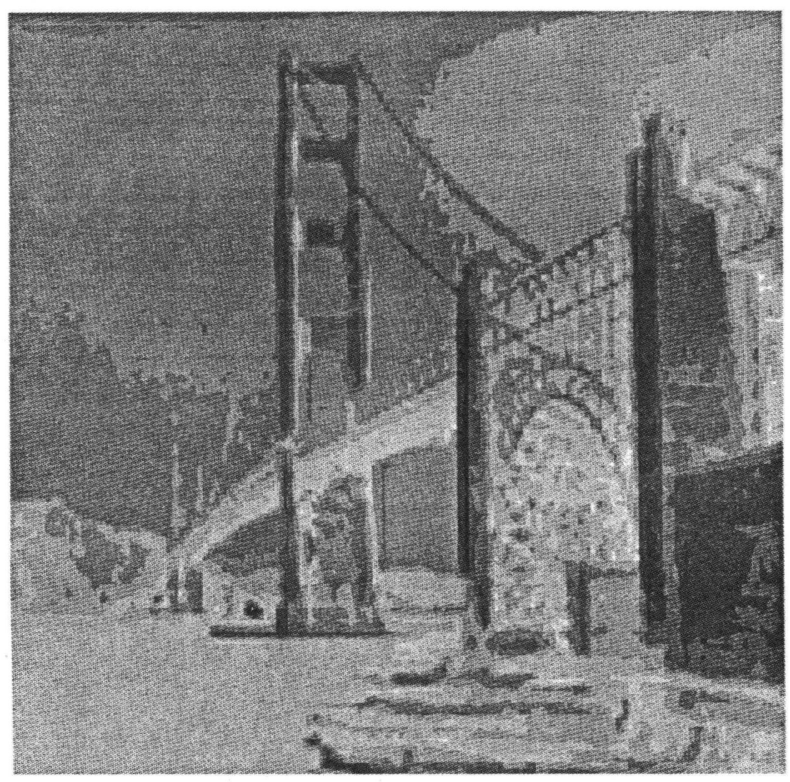

(a)

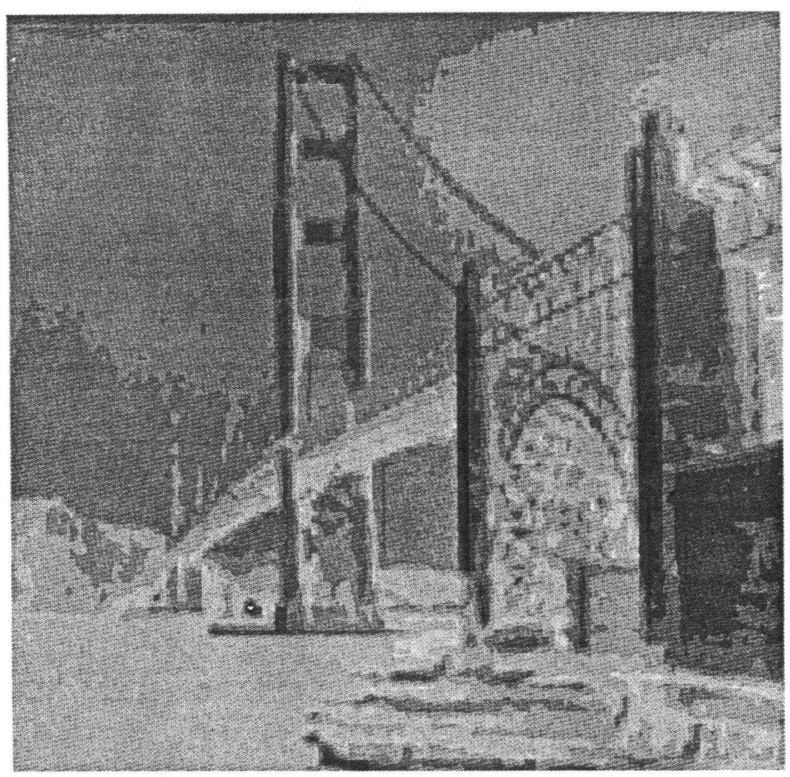

(c)

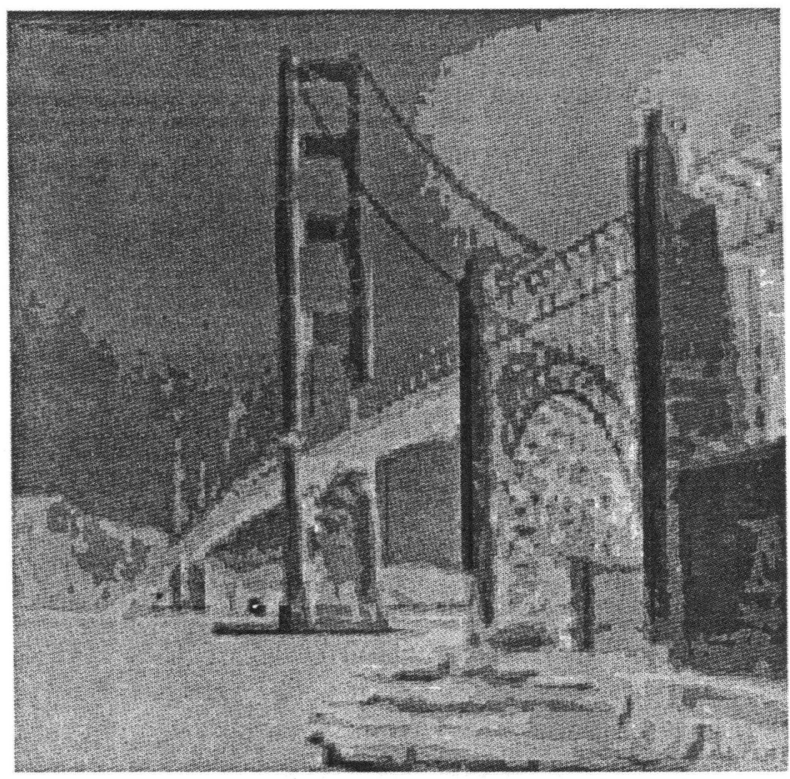

(b)

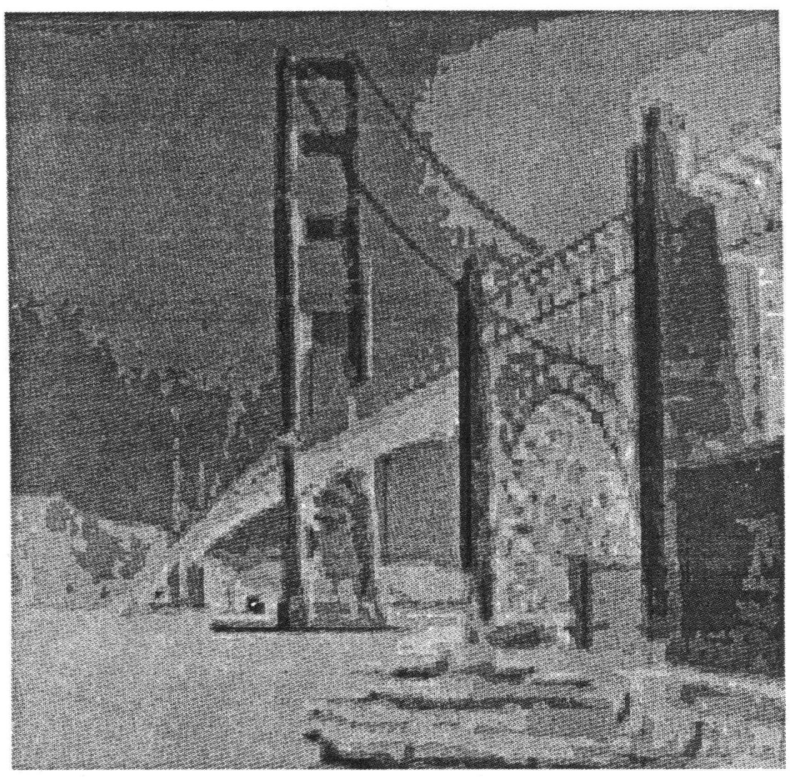

(d)

Fig. 8. Reconstructions of the picture of the Golden Gate Bridge. Block size: $8 \times 8$. Coefficients retained: $2 \times 2$. (a) IDCT reconstruction. (b) Regular MEP reconstruction. (c) First iteration of MEP-MREP reconstruction. (d) Second iteration of MEP-MREP reconstruction.

This is the first "iteration" of the proposed iterative algorithm. This solution has the proper form of the regular MREP solution of Eq. (3), and it satisfies the constraint of Eq. (20), but $\lambda_{0}$ may not be the proper value that satisfies the constraint of Eq. (19). (In the practical examples it was close.)

The second iteration is obtained as follows: The first iteration $\mathrm{f}_{1}(\mathrm{j})$ is used as the prior in minimizing the RE between $\mathrm{f}(\mathrm{j})$ and $f_{1}(j)$. The solution is denoted as $f_{0}^{\prime}(j)$ and is

$$
\begin{aligned}
\mathrm{f}_{0}^{\prime}(\mathrm{j}) & =\mathrm{f}_{1}(\mathrm{j}) \exp \left[-\lambda_{0} \mathrm{~g}_{1}(\mathrm{j})\right] \\
& =\mathrm{p}(\mathrm{j}) \exp \left[-\left(\lambda_{0}^{\prime}+\lambda_{0}\right) \mathrm{g}_{0}(\mathrm{j})-\lambda_{1} \mathrm{~g}_{1}(\mathrm{j})\right],
\end{aligned}
$$

This is inserted into the constraint of Eq. (19) to calculate the $\lambda_{0}$. If at this point $\lambda_{0}^{\prime}=0$, then the first iteration satisfies both constraints and thus is the correct solution to the regular MREP method. If not, the new $\lambda_{0}^{\prime}$ is found, and $\mathrm{f}_{0}(\mathrm{j})$ satisfies the first constraint but its $\lambda_{1}$ may not satisfy the second.

Next, the $f_{0}(j)$ serves as a prior and a new minimization is performed with Eq. (20) as a constraint. The new solution is

$$
\begin{aligned}
f_{1}^{\prime}(j) & =f_{0}^{\prime}(j) \exp \left[-\lambda_{1}^{\prime} g_{1}(j)\right] \\
& =p(j) \exp \left[-\left(\lambda_{0}+\lambda_{0}^{\prime}\right) g_{0}(j)-\left(\lambda_{1}+\lambda_{1}^{\prime}\right) g_{1}(j)\right],
\end{aligned}
$$

and $\lambda_{1}^{\prime}$ is calculated using the constraint of Eq. (20). This $f_{1}^{\prime}(j)$ represents the second "iteration" of the algorithm. It has the form of the regular MREP solution, and it satisfies the second constraint. However, the first constraint may not be satisfied, 


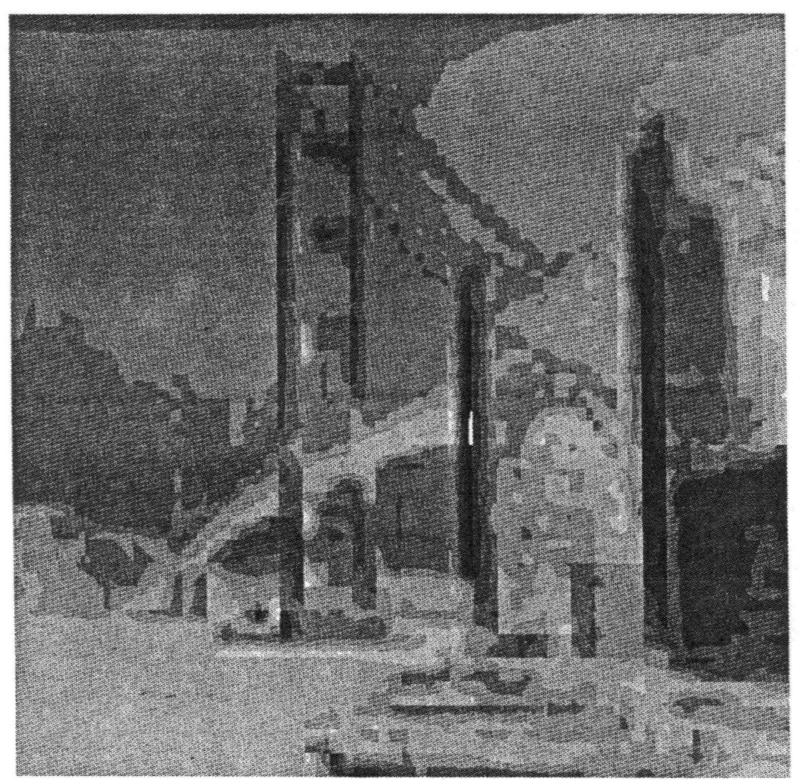

(a)

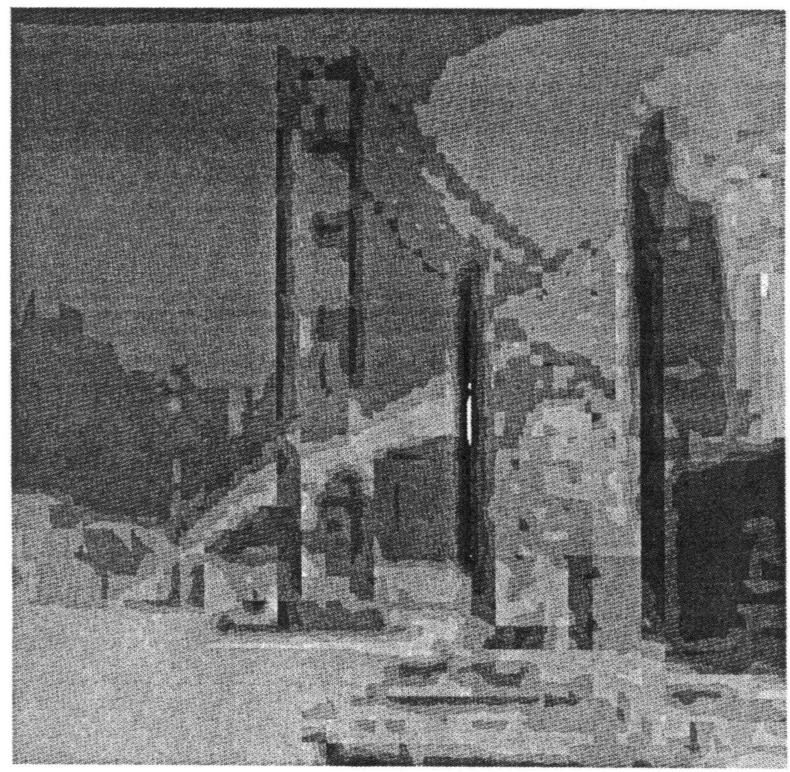

(c)

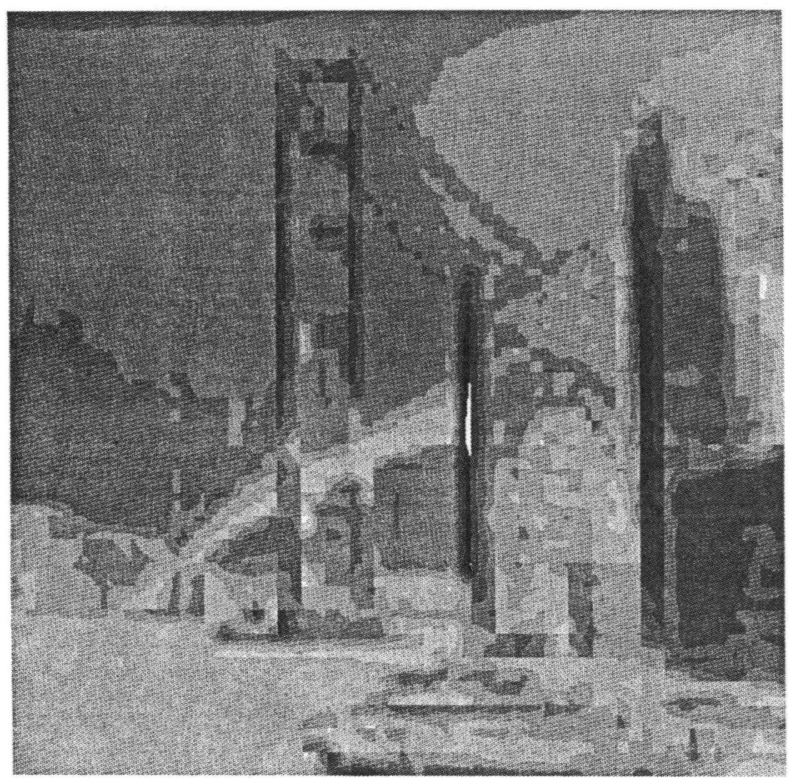

(b)

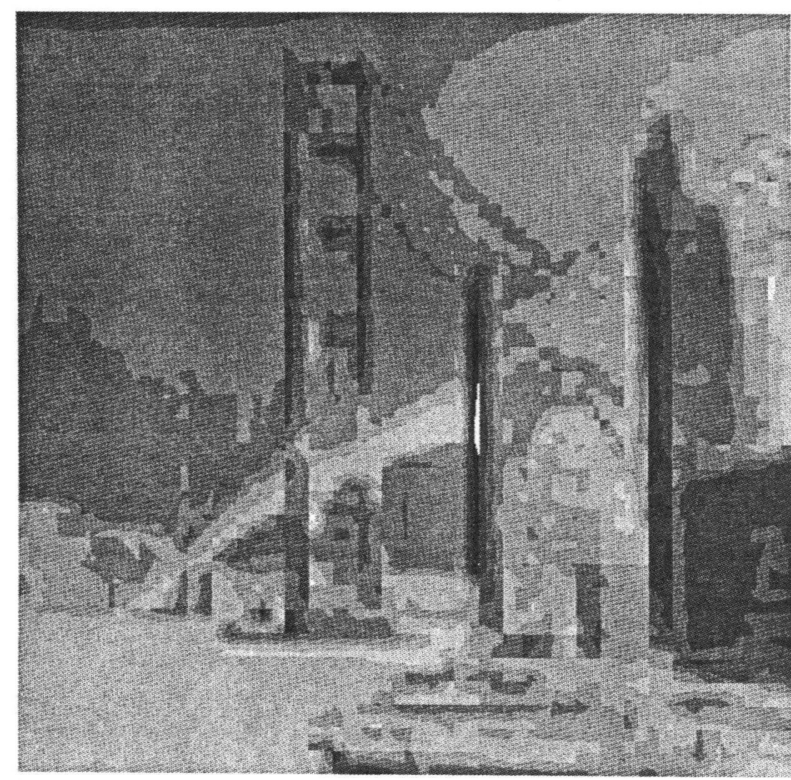

(d)

Fig. 9. Reconstructions of the picture of the Golden Gate Bridge. Block size: $16 \times 16$. Coefficients retained: $2 \times 2$. (a) IDCT reconstruction. (b) Regular MEP reconstruction. (c) First iteration of MEP-MREP reconstruction. (d) Second iteration of MEP-MREP reconstruction.

so a new passage through the constraint is initiated, leading to the third "iteration" of the algorithm, which will have the form

$$
\begin{gathered}
f_{1}^{\prime \prime}(j)=p(j) \exp \left[-\left(\lambda_{0}+\lambda_{0}^{\prime}+\lambda_{0}^{\prime \prime}\right) g_{0}(j)\right. \\
\left.-\left(\lambda_{1}+\lambda_{1}^{\prime}+\lambda_{1}^{\prime \prime}\right) g_{1}(j)\right] .
\end{gathered}
$$

It is clear from the above that if this solution converges (the $\lambda^{\prime}$ converge to zero), it will converge to the regular MREP solution of Eq. (3).

This type of iterative process has been demonstrated to converge for discrete distributions by Ireland and Kullback ${ }^{17}$ and for continuous distributions by Kullback. ${ }^{18}$ A more rigorous treatment of convergence, uniqueness, etc. appears in Csiszar. ${ }^{19}$ Experimentally, there appears little doubt that the algorithm converges and that it does so remarkably fast, usually at the second iteration.

\section{REFERENCES}

1. S. Kullback, Information Theory and Statistics, Dover, New York (1969).

2. J. E. Shore and R. W. Johnson, "Axiomatic derivation of the principle of maximum entropy and the principle of minimum cross-entropy," IEEE Trans. Inf. Theory IT-26, 26-37 (1980).

3. E. T. Jaynes, "Prior probabilities," IEEE Trans. Syst. Sci. Cybernet. SSC-4, 227-241 (1958).

4. J. E. Shore, "Minimum cross-entropy spectral analysis," IEEE Trans Acoust. Speech Signal Proc. ASSP-29(2), 230-237 (1981)

5. M. A. Tzannes, D. Politis, and N. S. Tzannes, "A general method of minimum cross-entropy spectral estimation,"IEEE Trans. Acoust. Speech Signal Proc. ASSP-33(3), 748-752 (1985).

6. J. P. Burg, "Maximum entropy spectral analysis," Ph.D. dissertation, Stanford Univ., microfilms No. 75-25, 499 (1975). 
7. A. K. Jain, "Advances in mathematical models for image processing," Proc. IEEE 69, 502-528 (1981).

8. S. F. Gull and J. Skilling, "The entropy of an image," in MaximumEntropy and Bayesian Methods in Inverse Problems, C. R. Smith and W. T. Grandy, eds., pp. 287-301, D. Reidel Publ. Co., Dordrecht, Netherlands (1985).

9. B. R. Frieden, "Restoring with maximum entropy and maximum likelihood," J. Opt. Soc. Am. 62(4), 511-518 (1972).

10. W. K. Pratt, Image Transmission Techniques, Academic Press, New York (1979).

11. N. S. Tzannes and P. A. Jonnard, "Maximum entropy reconstruction of moment-coded images," Opt. Eng. 26(10), 1077-1083 (1987).

12. A. J. Daniell and S. F. Gull, "Maximum entropy algorithm applied to image enhancement," IEE Proc. 127, Pt. F, No. 5, 170-172 (1980).

13. J. E. Shore, "State spaces and initial estimates in minimum relative entropy inversion with applications to spectrum analysis and image enhancement," Naval Research Laboratory report, Washington, D.C. (1984).

14. Y. Alsaka, N. S. Tzannes, and B. Marinelli, "An efficient algorithm for implementing the relative entropy method,'in Proc. 1988 Int. Conf. on Acoustics, Speech, and Signal Processing 2384-2387, IEEE (1988).

15. Y. Alsaka, N. S. Tzannes, and D. M. Gregory, "Multisignal spectral estimation using an iterative relative entropy method," in Proc. IEEE SOUTHEASTCON '88, 45-49 (1988).

16. K. Knowlton, "Progressive transmission of gray-scale and binary pictures by simple, efficient and lossless encoding schemes," Proc. IEEE 68(7), 885-896 (1980).

17. C. T. Ireland and S. Kullback, "Contingency tables with given marginals," Biometrika 55, 179-188 (1968).

18. S. Kullback, "Probability densities with given marginals," Ann. Math. Statist. 39, 1236-1243 (1968).

19. I. Csiszar, "I-divergence of probability distributions and minimization problems," Ann. Probability 3(1), 146-158 (1975).

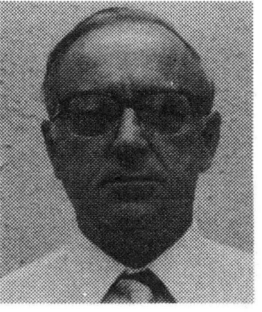

Nicolaos S. Tzannes is a professor in and chairman of the Department of Electrical Engineering and Communication Sciences at the University of Central Florida. He holds BSEE, MSEE, and Ph.D. degrees from the University of Minnesota, Syracuse University, and the Johns Hopkins University, respectively. Dr. Tzannes has taught at Johns Hopkins, Tufts University, the Universities of Virginia and Oklahoma, and the University of Patras, Greece. He is the author or editor of several books, the most recent being Communication and Radar Systems (Prentice-Hall, 1985). He also is the author or coauthor of more than 70 scientific papers on communications systems, digital signal processing, mathematical linguistics, etc., published in various journals or conference proceedings.

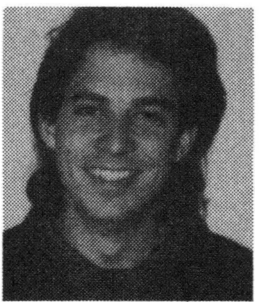

Michael A. Tzannes was born in 1961 in Minneaplis. He graduated valedictorian from the Electrical Engineering Deparment of the University of Patras, Greece, in 1984 and received an MSEE degree from the University of Michigan, Ann Arbor, in 1986. He then joined Signatron of Lexington, Mass., where he is working on analysis and software development for digital communication systems, over LOS and multipath fading channels. Some of the problems he is addressing concern efficient receiver structures, adaptive equalization algorithms, and main and auxilliary lobe jammer suppression. $\mathrm{He}$ is also currently working on new techniques to track multiple sonar/radar targets.

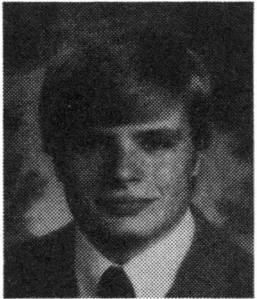

John S. Bodenschatz is currently doing research for the Department of Defense. $\mathrm{He}$ received his master's degree from the University of Central Florida in December 1987 and his bachelor's degree in electrical engineering from the University of Virginia in May 1986. 